\title{
An analysis of cloud overlap at a midlatitude atmospheric observation facility
}

\author{
L. Oreopoulos ${ }^{1}$ and P. M. Norris ${ }^{2,3}$ \\ ${ }^{1}$ Laboratory for Atmospheres, NASA-GSFC, Greenbelt, MD, USA \\ ${ }^{2}$ Global Modeling and Assimilation Office, NASA-GSFC, Greenbelt, MD, USA \\ ${ }^{3}$ GESTAR, University Space Research Association, Columbia, MD USA
}

Received: 7 December 2010 - Published in Atmos. Chem. Phys. Discuss.: 7 January 2011

Revised: 2 May 2011 - Accepted: 2 June 2011 - Published: 16 June 2011

\begin{abstract}
An analysis of cloud overlap based on high temporal and vertical resolution retrievals of cloud condensate from a suite of ground instruments is performed at a mid-latitude atmospheric observation facility. Two facets of overlap are investigated: cloud fraction overlap, expressed in terms of a parameter " $\alpha$ " indicating the relative contributions of maximum and random overlap, and overlap of horizontal distributions of condensate, expressed in terms of the correlation coefficient of condensate ranks. The degree of proximity to the random and maximum overlap assumptions is also expressed in terms of a decorrelation length, a convenient scalar parameter for overlap parameters assumed to decay exponentially with separation distance. Both cloud fraction overlap and condensate overlap show significant seasonal variations with a clear tendency for more maximum overlap in the summer months. More maximum overlap is also generally observed when the domain size used to define cloud fractions increases. These tendencies also exist for rank correlations, but are significantly weaker. Hitherto unexplored overlap parameter dependencies are investigated by analyzing mean parameter differences at fixed separation distance within different layers of the atmospheric column, and by searching for possible systematic relationships between alpha and rank correlation. We find that for the same separation distance the overlap parameters are significantly distinct in different atmospheric layers, and that random cloud fraction overlap is usually associated with more randomly overlapped condensate ranks.
\end{abstract}

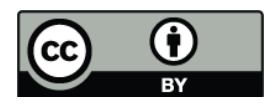

Correspondence to: L. Oreopoulos (lazaros.oreopoulos@ nasa.gov)

\section{Introduction}

While conspicuous, full 3-D cloud heterogeneity is generally ignored in atmospheric research applications. The underlying reasons for doing so include computational expediency, inability to diagnose or predict the heterogeneity, and insufficient understanding of how to meaningfully convey its impact on various atmospheric processes. In particular, although radiative transfer can in principle be accurately performed on a completely described 3-D cloud field, this capability cannot be trivially extended to Global Climate Models (GCMs). Thus far no clear pathway has been suggested to overcome challenges such as the unavailability of information on the nature and location of cloud edges, the lack of knowledge on how to make the resulting 3-D radiation fields relevant for other model processes, and the associated computational costs. Still, whereas specification of the horizontal spatial coherence necessary to describe true 3-D cloud fields appears to be beyond the capabilities of current GCMs, a goal that seems tenable with present modeling and observational capabilities is to describe the horizontal and vertical condensate variability of otherwise plane-parallel clouds using solely layer probability distributions and vertical correlations. Such an approach will probably be adequate for most radiative applications.

Recently, the coupling of cloud generators producing horizontal and vertical cloud variability with standard GCM radiative transfer algorithms operating stochastically has been suggested as a way to bypass direct incorporation of complex cloud structure in radiation schemes (Pincus et al., 2003). These cloud generators can also be used for pairing GCM cloud fields with simulators of instruments with much higher spatial resolution than the model grid size. To produce realistic one-point statistics of cloud condensate, and therefore radiation fields, both the horizontal variability and vertical correlations of cloud fraction and condensate distributions

Published by Copernicus Publications on behalf of the European Geosciences Union. 
need to be realistically described by the generators. This paper addresses the vertical correlations by providing a detailed examination of their characteristics in a dataset of 2-D distributions of condensate derived from a suite of ground-based instruments.

Our goal is to understand the features, dependencies, and intrinsic connections between two aspects of cloud vertical variability, overlap of cloud fraction and overlap of the horizontal distributions of cloud condensate. Ultimately, when studies such as this are completed for a more extensive range of cloud regimes, it may be possible to determine a simple but robust set of global rules that can be used to generate modeled clouds that resemble (save for horizontal spatial coherence) the original cloud fields and produce similar radiative fluxes and heating rates. While coarser resolution measurements similar to those used here have been previously analyzed in studies of cloud fraction overlap (from ground or space), condensate distribution overlap and its relationship with cloud fraction overlap has not been studied before with an observationally-based dataset.

\section{Dataset, definitions, and overlap metrics}

Our overlap analysis relies exclusively on the continuous baseline microphysical retrieval MICROBASE evaluation product (Miller et al., 2003) of the Atmospheric Radiation Measurement (ARM) Climate Research Facility (ACRF), now part of the US Department of Energy Atmospheric System Research (ASR) Program. The MICROBASE retrieval algorithm uses a combination of observations from a millimeter cloud radar (MMCR), a ceilometer, a micropulse lidar (MPL), a microwave radiometer (MWR), and balloonborne sounding profiles to estimate the profiles of liquid/ice water content (LWC/IWC), liquid/ice cloud particle effective radius, and cloud fraction. For liquid cloud layers (atmospheric temperatures greater than $273 \mathrm{~K}$ ) MICROBASE uses the radar reflectivity-LWC relationship derived by Liao and Sassen (1994). The LWC profile is vertically integrated to provide a liquid water path (LWP) which is then linearly scaled to match the LWP observed by the MWR. For atmospheric temperatures below $257 \mathrm{~K}$ all water is assumed to be in the ice phase, and its content is determined using the radar reflectivity-IWC relationship of Liu and Illingworth (2000). Between 257 and $273 \mathrm{~K}$ water is assumed to exist in both phases and a linear temperature-dependent partition of ice/liquid is applied. The radar reflectivities used in the above relationships come from the Active Remote Sensing of Clouds (ARSCL) product (Clothiaux et al., 2000). While particle size retrievals are also performed as part of the MICROBASE algorithm, they are not used in the present study. Cells that are flagged to have no reflectivity data are identified in MICROBASE and are discarded from the analysis.

The MICROBASE data of this study are for the Southern Great Plains (SGP) ACRF site in Oklahoma, USA (http://www.arm.gov/sites/sgp). The dataset spans seven years (2000-2006) and data availability, although not uniform, covers all 84 months. The 2-D condensate distribution is available at a $10 \mathrm{~s}$ resolution along the advection path of the clouds over the instruments, and $45 \mathrm{~m}$ vertical resolution (constrained by the MMCR range gate). For the purposes of this study, the condensate profiles for each day are divided into segments that roughly correspond to scales of typical GCMs. For example, when six segments are used per day, each segment consists in general of 1440 condensate profiles, which correspond to scales of $\sim 150 \mathrm{~km}$ assuming typical wind speeds of $10 \mathrm{~m} \mathrm{~s}^{-1}$. These 1440-profile segments are our default choice for the overlap analysis, with 720- and 2880-profile segments used only when we want to highlight the sensitivity of an overlap metric to the pseudo-spatial reference scale. Implicitly assuming a one-to-one correspondence between temporal and spatial domains while common (see for example Hogan and Illingwoth, 2000), is obviously an approximation. It does not account for changes in wind speed with time and height, factors that can be more easily ignored in radar observations from space where the instrument moves at a high speed and in a direction not generally aligned with that of cloud advection. Our adoption of the so-called "frozen turbulence" assumption and of a constant wind speed, while not optimal or fully realistic, is designed to provide an easier interpretation of our results for GCM parameterizations.

Our analysis does not distinguish between the liquid and ice phases, but rather operates on the total water content, i.e., the sum of LWC and IWC. For our cloud fraction overlap analysis we calculate the true combined segment cloud fraction $C_{t}\left(z_{1}, z_{2}\right)$ of a pair of layers separated by distance $\Delta z=z_{2}-z_{1}-$ where $z_{2}$ and $z_{1}$ are the heights of the layer centers as determined by the vertical resolution of the dataset, so that $\Delta z$ is always a multiple of $45 \mathrm{~m}$ - by counting among the pairs with cells flagged as having valid (zero or non-zero) retrievals at both heights those that have non-zero total water content at one or both two height levels of interest and dividing by the total number of such pairs. Individual layer cloud fractions $C\left(z_{1}\right)$ and $C\left(z_{2}\right)$ are calculated by dividing the number of cloudy (total water content greater than zero) cells in each layer by the same number of valid profiles as in the calculation of $C_{t}\left(z_{1}, z_{2}\right)$. From the individual layer cloud fractions, combined cloud fractions corresponding to the maximum and random overlap assumption can be calculated as follows:

$$
\begin{aligned}
& C_{\max }\left(z_{1}, z_{2}\right)=\max \left(C\left(z_{1}\right), C\left(z_{2}\right)\right) \\
& C_{\text {ran }}\left(z_{1}, z_{2}\right)=1-\left(1-C\left(z_{1}\right)\right)\left(1-C\left(z_{2}\right)\right)
\end{aligned}
$$

Hogan and Illingworth (2000) proposed that the combined cloud fraction of two layers can be approximated as a weighted average of $C_{\max }\left(z_{1}, z_{2}\right)$ and $C_{\mathrm{ran}}\left(z_{1}, z_{2}\right)$ according to:

$C\left(z_{1}, z_{2}\right)=\alpha\left(z_{1}, z_{2}\right) C_{\max }\left(z_{1}, z_{2}\right)+\left(1-\alpha\left(z_{1}, z_{2}\right)\right) C_{\mathrm{ran}}\left(z_{1}, z_{2}\right)(2)$ 
When $C_{t}\left(z_{1}, z_{2}\right)$ is known, as in our case, it can be substituted in the left hand side of the above equation to obtain the weighting parameter $\alpha\left(z_{1}, z_{2}\right)$, a measure of the proximity of overlap to maximum (exact when $\alpha\left(z_{1}, z_{2}\right)=1$ ) or random (exact when $\alpha\left(z_{1}, z_{2}\right)=0$ ). Negative values suggest some degree of minimum overlap (a combined cloud fraction greater than that of random overlap). Without distinguishing between contiguous and non-contiguous cloud layers, we calculate $\alpha\left(z_{1}, z_{2}\right)$ for our entire dataset for each possible cloud fraction pair for separation distances ranging from 45 to $12015 \mathrm{~m}$ (1 to 267 layer separations) as long as neither of the cloud fractions is zero or one. This procedure yields a very large dataset of $\alpha\left(z_{1}, z_{2}\right)$ values which we then segregate by month. The number of valid $\alpha\left(z_{1}, z_{2}\right)$ values within a month over 7 years can exceed 7 million for $150 \mathrm{~km}$ segments. In the following we frequently refer to this parameter simply as "alpha".

In a similar fashion, we calculate rank correlations of total water content as a function of separation distance (see also Pincus et al., 2005). For layers at heights $z_{1}$ and $z_{2}$, the overlapping cloudy cells are identified (i.e., non-zero total water contents in both layers), and their water contents are ranked at each height. A linear correlation coefficient $r\left(z_{1}, z_{2}\right)$ is then calculated from the ranks $R_{i}\left(z_{1}\right)$ and $R_{i}\left(z_{2}\right)$ according to:

$$
r\left(z_{1}, z_{2}\right)=\frac{\sum_{i=1}^{N_{\text {cld }}}\left(R_{i}\left(z_{1}\right)-\bar{R}\left(z_{1}\right)\right)\left(R_{i}\left(z_{2}\right)-\bar{R}\left(z_{2}\right)\right)}{\sqrt{\sum_{i=1}^{N_{\text {cld }}}\left(R_{i}\left(z_{1}\right)-\bar{R}\left(z_{1}\right)\right)^{2}} \sqrt{\sum_{i=1}^{N_{\text {cld }}}\left(R_{i}\left(z_{2}\right)-\bar{R}\left(z_{2}\right)\right)^{2}}}
$$

where $N_{\text {cld }}$ is the number of overlapping cells and $\bar{R}\left(z_{1}\right)$, $\bar{R}\left(z_{2}\right)$ are the mean ranks of those $N_{\text {cld }}$ water contents in the two layers. The rank correlation coefficient expresses the likelihood water contents of the same relative strength within their respective layers are aligned in the vertical, with $r\left(z_{1}, z_{2}\right)=1$ corresponding to perfect alignment and $r\left(z_{1}, z_{2}\right)=0$ corresponding to completely random alignment.

Unlike $\alpha\left(z_{1}, z_{2}\right)$ calculations, overcast layers are not excluded for the rank correlations. It should also be pointed out that since the overlapping portion changes continuously with the pairing partner, the part of a specific layer being ranked is in general different for each rank correlation calculation. In other words, ranks are calculated anew as dictated by the common portion of the two layers. The manner in which water contents align in the vertical can be important for processes like radiation. For example, the domain-averaged radiative fluxes differ between a case where all high or low condensate values are aligned to create pockets of vertically integrated high or low water paths and a case where a more random alignment homogenizes the horizontal distribution of water path (e.g., see Norris et al., 2008). The full dataset of all possible $r\left(z_{1}, z_{2}\right)$ values is derived from MICROBASE condensate for the period 2000-2006 in a manner similar to alpha, as described above, including segregation by month.
It has been suggested (e.g., Hogan and Illingworth, 2000; Pincus et al., 2005; Shonk et al., 2010) that profiles of alpha and rank correlation can be modeled as inverse exponential functions

$\alpha(\bar{h}, \Delta z)=\exp \left(-\frac{\Delta z}{L_{\alpha}(\bar{h})}\right)$

$r(\bar{h}, \Delta z)=\exp \left(-\frac{\Delta z}{L_{r}(\bar{h})}\right)$

where $L_{\alpha}$ and $L_{r}$ are decorrelation length scales which can be viewed as alternate measures of the degree of overlap. Specifically, large values of $L_{\alpha}$ indicate proximity to maximum overlap, while small values proximity to random overlap. Likewise, large values of $L_{r}$ indicate condensate values that are highly correlated in terms of relative strength while small values indicate condensate values whose relative strength exhibits weak correlation between layers. In Eq. (4) an explicit dependence of the overlap parameters and decorrelation lengths on the mean height $\bar{h}$ of the atmospheric layer in which they are calculated is assumed. This is intended to convey the notion that identical separation distances may give rise to systematically different overlap behavior in various vertical locations of the atmosphere where different cloud formation processes and dynamical characteristics are encountered. One of the drawbacks of inverse exponential modeling is that negative values of the overlap parameters cannot be captured (Norris et al., 2008). This turns out to be a poorer approximation for the condensate rank correlation, for which negative values are encountered much more frequently, than alpha. In the analysis that follows, overlap is discussed both in terms of the overlap parameter alpha and the rank correlation as well as in terms of their respective decorrelation lengths. Decorrelation lengths offer the convenience of a simple scalar representation of overlap, while alpha and rank correlation arrays contain more details on the potentially complex full dependence on layer height pairs $\left(z_{1}, z_{2}\right)$.

\section{Overlap characteristics at the SGP ACRF site}

\subsection{Seasonal cycle of overlap parameters and their decorrelation lengths}

To derive the monthly profiles of $\alpha(\Delta z)$ and $r(\Delta z)$ we calculate the ensemble average for each month all values of $\alpha\left(z_{1}, z_{2}\right)$ and $r\left(z_{1}, z_{2}\right)$ that have the same separation distance $\Delta z$. The number of values that enter this calculation decreases monotonically with separation distance. For now, we do not distinguish between $\Delta z$ 's at different levels of the atmosphere, although we will examine this dependence later. The monthly values can be further averaged to seasonal averages for winter (DJF), spring (MAM), summer (JJA) and fall (SON). Figure 1 shows seasonal averages of alpha and Fig. 2 

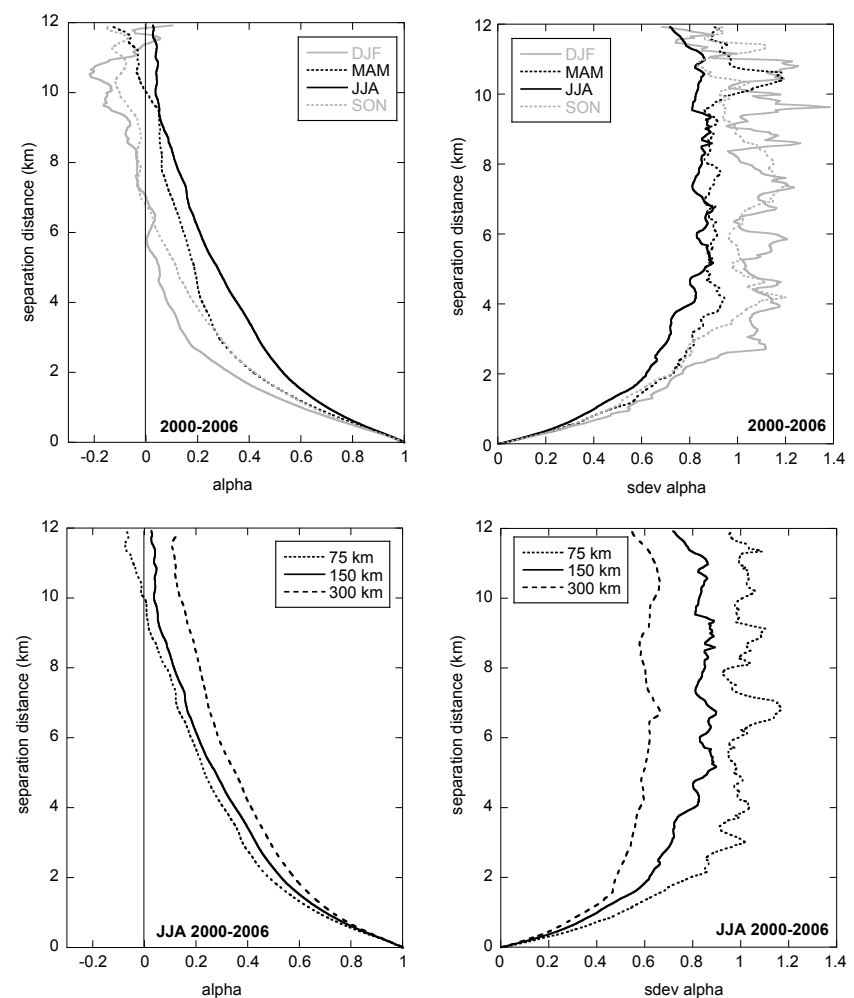

Fig. 1. Profiles of seasonal ensemble means (left panels) and standard deviations (right panels) of the overlap parameter alpha as a function of layer separation distance calculated from the 7-year MICROBASE dataset. Top panels are for the $150 \mathrm{~km}$ segment size and all four seasons: December-January-February (DJF, winter), March-April-May (MAM, spring), June-July-August (JJA, summer), September-October-November (SON, fall). Bottom panels are for different segment sizes for JJA.

shows seasonal averages of rank correlation; the profiles of standard deviation for both quantities are also provided in separate plots. The figures show both the seasonal dependence for a given segment size $(150 \mathrm{~km})$ and the dependence on segment size for a given season (JJA was chosen-the dependence is similar for other seasons). As clarified earlier, the three segments sizes, $75,150,300 \mathrm{~km}$, should not be considered as actual segment spatial scales, but as being roughly equivalent to a fixed number of 720,1440 , and 2880 condensate profiles.

In the analysis that follows, we will focus mainly on the description of the characteristics of overlap as extracted from the dataset and will not consistently attempt to provide an interpretation of the underlying reasons behind the overlap features that emerge. Such interpretations are often not obvious and would require extensive additional meteorological data not provided in the MICROBASE dataset. A comparison of Figs. 1 and 2 indicates that alpha profiles vary more with season and domain size than rank correlation profiles. They also drop much more slowly with separation distance compared to rank correlations. The decrease of alpha with separation
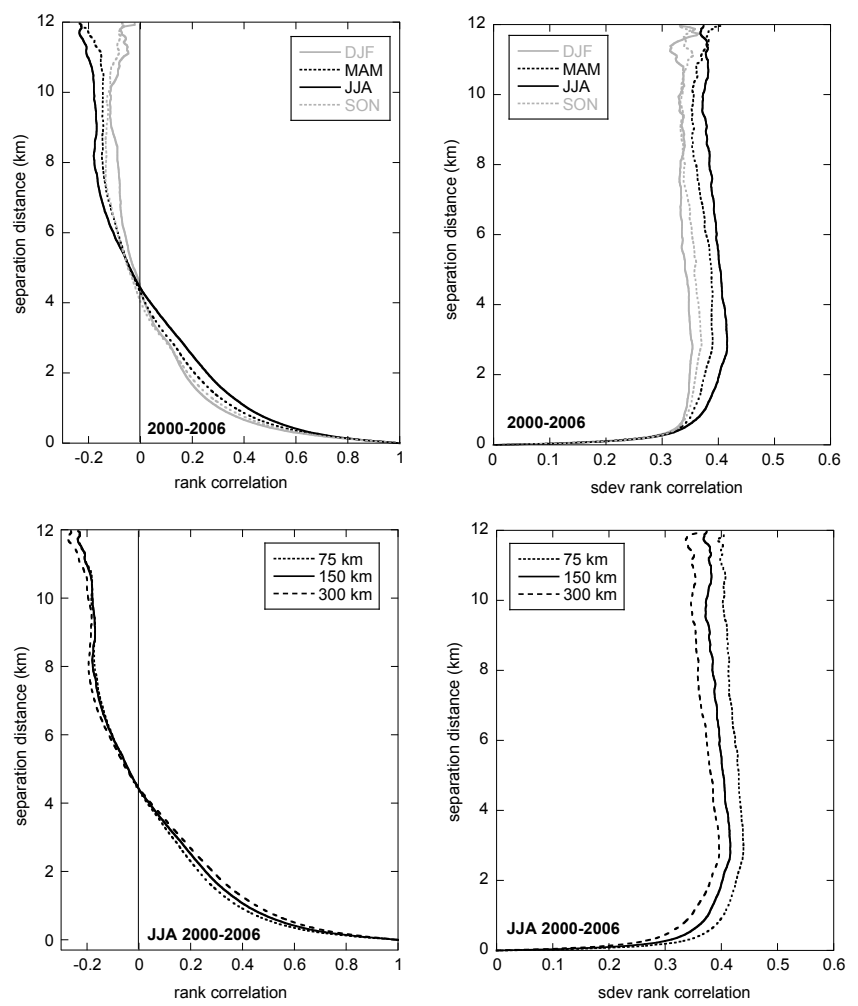

Fig. 2. As in Fig. 1, but for rank correlations.

distance is faster for winter, followed by fall, spring and summer. In other words, cloud fraction overlap is most random in the winter and least random (most maximum) during the summer. Since convective activity is greatest during the summer while winter cloudiness is dominated by frontal systems, the conclusion is that convective clouds are more maximally overlapped than frontal clouds. This was also found by Mace and Benson-Troth (2002) and Naud et al. (2008). The first of these papers showed the seasonal cycle of alpha at select separation distances over the same observation site, but from a data set of coarser temporal and vertical resolution derived independently from the same suite of instruments used in MICROBASE.

Our results also indicate that the variability (standard deviation) of alpha profiles follows in general the order of degree of random overlap: the alpha profile with the smallest values (DJF) is also the most variable; during summer the alpha values are larger (more maximally overlapped) and the distribution of alpha values is more narrow. This seems reasonable - if random overlap is produced by independent clouds layers at various heights, then we expect to get many cases of chance alignments between layers on a per segment basis, thereby injecting a random element of "maximum overlap" and increasing the variance of alpha. In contrast, maximum overlap cases produced by convective systems with strong vertical coherence are not expected to produce random overlap by chance, unless there is a strong vertical wind shear. 
The choice of domain size affects the alpha profiles noticeably. Cloud fraction overlap is more maximum for the largest domain size $(300 \mathrm{~km})$. This has been previously noted by Hogan and Illingworth (2000) and Oreopoulos and Khairoutdinov (2003) and is the natural outcome of the dominant scales of cloud formation as determined by underlying dynamical and thermodynamical processes. Indeed, for isolated cloud systems the chance of finding large total cloud fractions decreases as the domain size increases, and since random overlap means larger total cloud fractions than maximum overlap for the same cloud fraction profile, the overlap will tend to be more random within a smaller domain. Another thought experiment that leads to the same conclusion - that the degree of overlap is smaller (more random) for a smaller domain size - is to consider a particular cloud fraction profile within a certain domain. By enlarging the domain without changing the cloud (whose spatial extent is determined by the dominant scales of the underlying dynamics and thermodynamics) both the layer cloud fraction and the total cloud fraction decrease (layer clear fractions and total clear fraction increase). The cloud system occupies a relatively smaller portion of the bigger domain and cloud layers appear more aligned (more maximally overlapped) in the vertical since the combined clear fraction of any two layers has increased.

This type of argument does not carry over trivially to rank correlations which seem to also show the same dependence, albeit significantly weaker, on domain size. At larger domain sizes the probability density function of condensate must in general become wider and the relative ordering of condensate values must change so that the values of particular portions of the domain with more similar clouds are closer in relative strength compared to the case where the domain is smaller and the inter-layer variability in those same portions appears larger. In other words, by extending the domain and widening the distribution with the additional variability of extra clouds, the values of condensate at close horizontal positions appear more similar in a relative sense than in the initial narrower distributions.

The seasonal ordering in terms of the magnitude of rank correlation profiles is the same as for alpha profiles for separation distances up to $\sim 4 \mathrm{~km}$ where positive values occur. Rank correlations are generally smaller for DJF and progressively increase for MAM and JJA before dropping again for SON. This is consistent with stronger vertical motions during the summer producing more aligned columns of cloud condensate. However, the picture reverses for the negative rank correlations of larger separation distances which are greater in absolute value for JJA and smaller (closer to zero) for DJF. Apparently the low and high clouds of summer multilayer cloud systems are more anticorrelated than in the winter. Since the negative values of alpha do not exhibit such reversal, i.e., DJF cloud fractions are more minimally overlapped than JJA, the conclusion is that for the smaller overlapped portion of DJF clouds the anticorrelations of relative condensate strengths are somewhat weaker. As we will see in the next section, however, when all separation distances and seasons are ensemble-averaged there is a clear tendency for smaller alphas to be correlated with smaller rank correlations. This is not surprising since this is exactly the tendency that Figs. 1 and 2 imply at smaller separations, which are derived from a much larger number of data points. It should also be kept in mind that the condensate dataset used for Figs. 1 and 2 is not exactly identical since overcast layers are excluded from the calculation of alpha but not of rank correlation.

Another difference in the behavior of rank correlations is that the variability of rank correlations is least in DJF and greatest in JJA, i.e., the opposite of what takes place for alphas. This is somewhat expected given that the mean profile of rank correlation, itself coming from a wide distribution of segment-length rank correlations, is more extreme in an absolute sense for JJA (more positive at smaller separations, more negative at larger separations) than DJF. The variability stabilizes to near-constant values at or above smaller separation distances, $\sim 2 \mathrm{~km}$ or below depending on the season, compared to alpha variability which becomes more stable (apart from the superimposed noise of the smaller sample size) only at separation distances above $\sim 3 \mathrm{~km}$. In conclusion, for both alpha and rank correlations, the variability increases rapidly up to a certain separation distance and then changes more slowly. Also, the variability that the parameters settle to is much larger $(0.8-1)$ for alpha, compared with the rank correlation (0.35-4). It must be pointed out however that precise descriptions of the behaviour of alpha or rank correlation at large distances may not in practice be crucial. For example, in radiative calculations, distant layers are at most times radiatively uncoupled because of the high likelihood of substantial amounts of intervening cloud. In this case radiative transfer would be largely insensitive to the cloud fraction and condensate rank overlap. Moreover, for clouds separated by large distances, radiation that has interacted with one cloud layer has diffused much by the time it reaches the other layer, further reducing the importance of the details of cloud fraction and rank overlaps.

The ensemble-averaged alpha and rank correlation profiles of individual months (not shown) can be fit to inverse exponentials via least squares, following chapter 15.2 of Press et al. (1992), in order to infer the decorrelation lengths of Eq. (4). The calculations assumes no dependence on the vertical location $\bar{h}$ within the atmosphere (i.e., no distinction is made between equal $\Delta z$ 's in different parts of the atmosphere). The fitting gives greater weight to smaller separation distances which are more numerous. The results for the different segment lengths are given in Fig. 3, as a function of the month of the year. The figure reflects some of the seasonal and spatial scale dependencies discussed previously, for example decorrelation lengths that peak during the summer months when vertical stability is expected to be weaker and stronger vertical motions promote the formation of cloud 
systems with cloud fractions and condensates that align better. Alpha decorrelation lengths are larger than their rank correlation counterparts, with a stronger seasonal cycle and more pronounced dependence on domain size, echoing the contrasts we highlighted in our discussion of Figs. 1 and 2.

We also calculated, but do not show here, the median values of the decorrelation lengths derived for each segment and each month, as in Barker (2008b). The profiles of the overlap parameters for each individual segment are much more noisy and the fits much less reliable. Decorrelation lengths of alpha for individual segments can be very large, as also noted by Barker (2008a) (they exceed $10 \mathrm{~km} 36.5 \%$ of the time), making the mean values of limited use, and skewing the medians to values much higher (about double) than those calculated from ensemble-averaged overlap parameter profiles. For the rank correlation decorrelation lengths of individual segments, however, large magnitudes are much rarer (only for $\sim 1 \%$ of the cases exceed $10 \mathrm{~km}$ ) and the range of values is much narrower. The histograms of the two decorrelation length distributions for all $150 \mathrm{~km}$ segments of all months, but without the values greater than $10 \mathrm{~km}$, are compared in Fig. 4. The $L_{\alpha}$ istogram is much wider, has no well-defined peak, and looks quite different from the $L_{r}$ histogram which peaks at the $0.2-0.4 \mathrm{~km}$ bin. Despite the fact that the mode of the latter histogram is very small, the mean derived from the histogram, $1.74 \mathrm{~km}$, is larger than any of the values shown in Fig. 3, and serves as a reminder that the mean of decorrelation lengths derived from individual segments is a fundamentally distinct quantity from the decorrelation length derived from a mean profile of rank correlations. This is even more true for alpha decorrelation length, which has an even wider distribution in Fig. 4.

\subsection{Dependence of overlap parameters on vertical location}

In our earlier discussion of Eq. (4) we suggested that identical separation distances may give rise to systematically different overlap parameter values in different vertical segments of the atmosphere due to distinct cloud formation processes and associated dynamical circulations. In this subsection we examine whether this can indeed be discerned from the available dataset. Figure 5 shows ensemble-averaged alphas and rank correlations at separation distances of 1 and $2 \mathrm{~km}$ aggregated separately for four different atmospheric layers. The error in the mean is too small to be distinguishable in these plots and is therefore not shown, ensuring that any differences among the means are statistically significant. For alpha, there is a general trend towards more random overlap for the same separation distance the higher the atmospheric layer in which the calculation performed. This is always true for the $2 \mathrm{~km}$ separation distance, but not so for the $1 \mathrm{~km}$ separation distance as one moves from the $0-3 \mathrm{~km}$ layer to the 3-6 km layer. The rank correlation behavior, on the other hand, is somewhat more complex. The $0-3 \mathrm{~km}$ layer has the

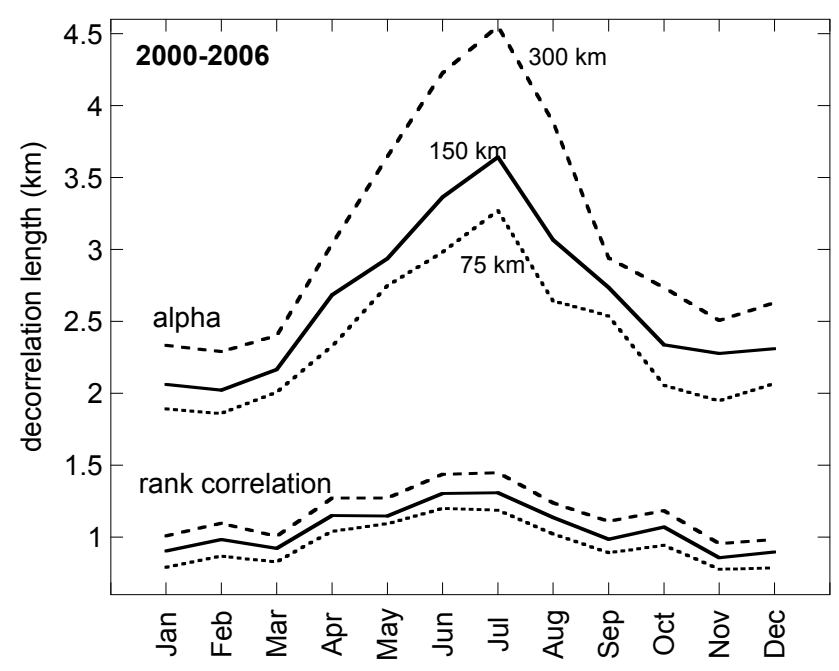

Fig. 3. The seasonal cycle of $L_{\alpha}$ (top three curves) and $L_{r}$ (bottom three curves) decorrelation lengths from ensemble-averaged profiles of alpha and rank correlation like those shown in Figs. 1 and 2, but on a monthly instead of tri-monthly temporal scale. The $L_{r}$ curves are ordered with segment size in the same manner as the $L_{\alpha}$ curves.

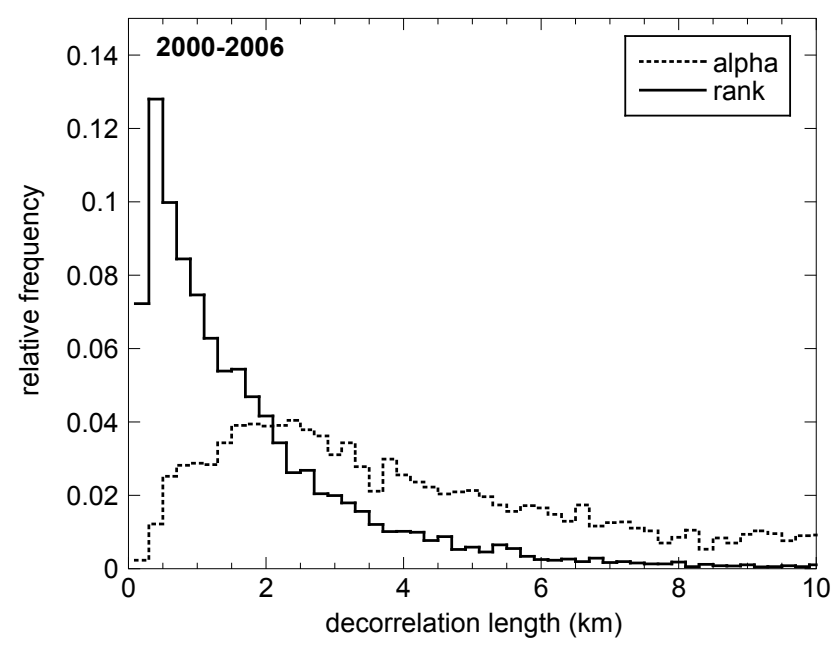

Fig. 4. Histograms of $L_{\alpha}$ and $L_{r}$ derived from individual $150 \mathrm{~km}$ segments. Due to the existence of a large fraction of $L_{\alpha}$ values greater than $10 \mathrm{~km}$ (indicative of near-maximum overlap conditions), both histograms were renormalized with such values excluded.

largest values at both separation distances, while the smallest are encountered in the 3-6 km layer for the $2 \mathrm{~km}$ separation distance and the 9-12 km layer for the $1 \mathrm{~km}$ separation distance. The large decrease of the rank correlation from the $0-3 \mathrm{~km}$ to the $3-6 \mathrm{~km}$ layer can probably be attributed to the cloud phase change that likely occurs within the latter layer and to the transition from the planetary boundary layer, which tends to be more well-mixed, to the free troposphere, which tends to be more stratified. The probability of both of 

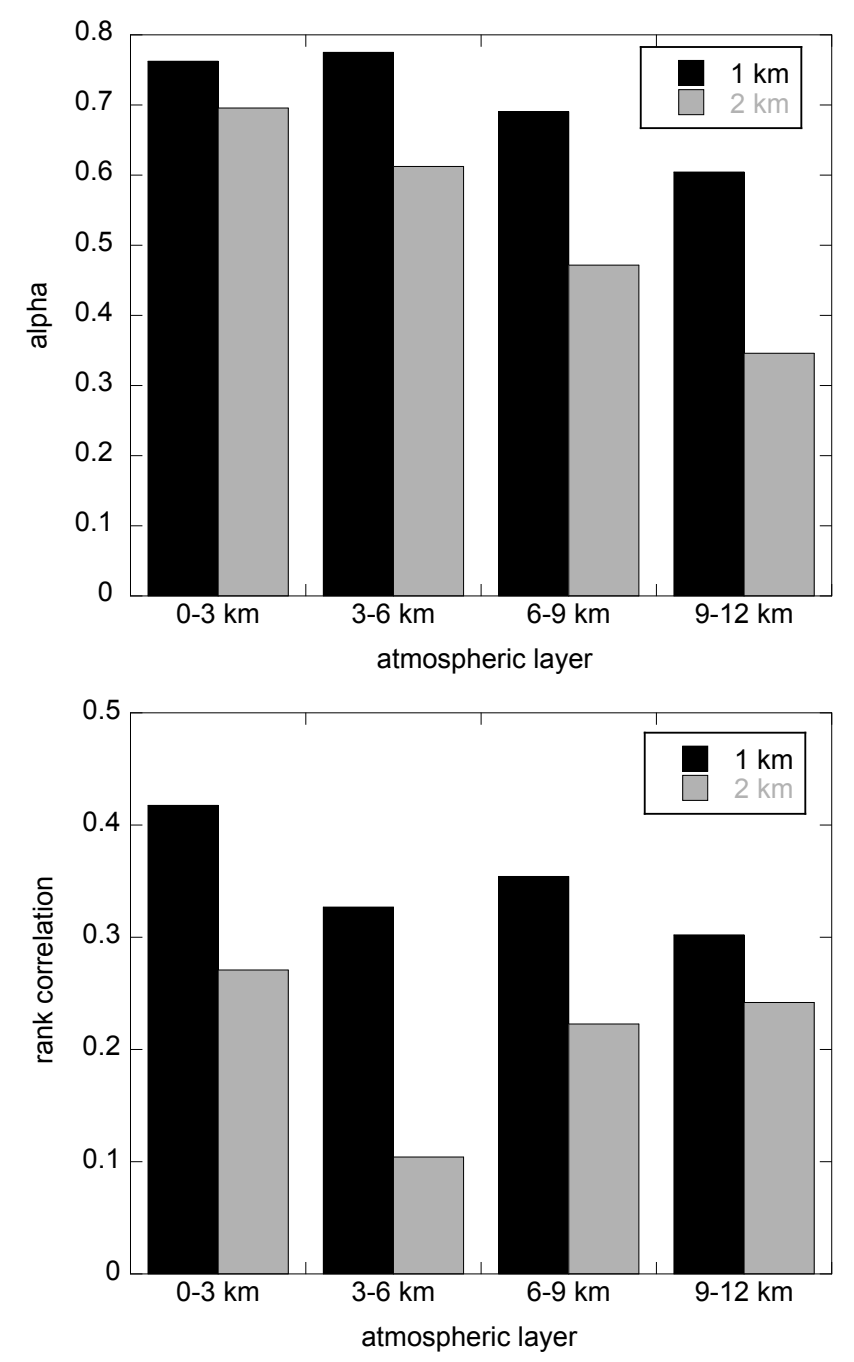

Fig. 5. Alphas (top) and rank correlations (bottom) for the $150 \mathrm{~km}$ segment size and separation distances of 1 and $2 \mathrm{~km}$ when ensemble-averaged separately within four different $3 \mathrm{~km}$ thick atmospheric layers. $0-3 \mathrm{~km}$ corresponds to the atmospheric layer closer to the surface.

these transitions occurring is greater for $2 \mathrm{~km}$ separation distances, which may be the reason for the observed minimum in rank correlation. Hogan and Illingworth (2003) examined the linear correlations of ice water content for overcast clouds above and below $6.9 \mathrm{~km}$. They found greater correlations in the upper layer, a result they attributed to the reduced wind shear of the upper layer and which is qualitatively similar to the increase of rank correlation from the 3-6 km layer to the $6-9 \mathrm{~km}$ layer in our results. The datasets and methodology are different enough to prevent us from affirming consistency between the two findings, but the qualitative agreement is nonetheless worth mentioning. Naud et al. (2008) also studied the role of wind shear on cloud overlap but for cloud fraction only, i.e., the effect on alphas, not rank correlations. They found higher wind shear correlating with smaller alphas above $\sim 2 \mathrm{~km}$ separation distances. If shear was the sole dynamical factor regulating cloud overlap then our results would seem to imply that shear must increase with height since according to Fig. 5 cloud fraction overlap tends to be generally more random in the upper troposphere compared to the lower troposphere. In our case, such an interpretation can not be provided with confidence based on the available information.

\subsection{Relationship between overlap parameters}

If the alpha and rank correlation overlap parameters are to be used to generate columns of condensate that follow the overlap behavior seen in observations, it may not be wise to choose their values independently of each other. In a modeling application, the most convenient approach would be to deal with scalar quantities such as the decorrelation lengths of exponentially decaying alphas and rank correlations and accepting the (hopefully minor) shortcoming of being confined to positive-only values. A plot like Fig. 3 can be employed to select $L_{\alpha}$ and $L_{r}$ values that can then be used at all times for each month at the appropriate latitudes and domain sizes. This plot implies that the ratio of $L_{\alpha}$ to $L_{r}$ changes substantially from month to month (from a minimum of $\sim 2$ in February to a maximum of $\sim 2.8$ in July and September). The appropriateness of picking a single value of $L_{\alpha}$ and (independently or not) of $L_{r}$ and applying it universally for a particular month will presumably depend on the application and should be a matter for further investigation, as will be discussed in the next section.

If one wants to discuss relationships between the two types of overlap, it may not however be appropriate to compare only quantities derived after a large amount of ensemble averaging, which is the approach yielding meaningful values of decorrelation lengths. We will therefore revisit segmentlevel alphas and rank correlations for our investigation of the relationship between cloud fraction and condensate distribution overlap. We will also investigate whether rank correlations depend on the combined cloud fraction of two layers. This fraction should not be independent of alpha since for a given pair of cloud fractions, a smaller alpha implies a larger combined cloud fraction. So, while we may get a somewhat different perspective by looking at how ranks change with different combined cloud fractions, that perspective cannot be inconsistent with that obtained by looking at rank correlation vs. alpha relationships.

In order to examine these relationships both overlap parameters need to be derived from the same data set. Since an alpha value is not meaningful when one of the two layers is overcast while a rank correlation is, for the purposes of this subsection we infer both overlap parameters only when neither of the two layers has a cloud fraction greater than 0.99 while also imposing the condition that the overlapped portion of the two layers has a cloud fraction of at least 0.01 (to have enough data points for an acceptable rank correlation 

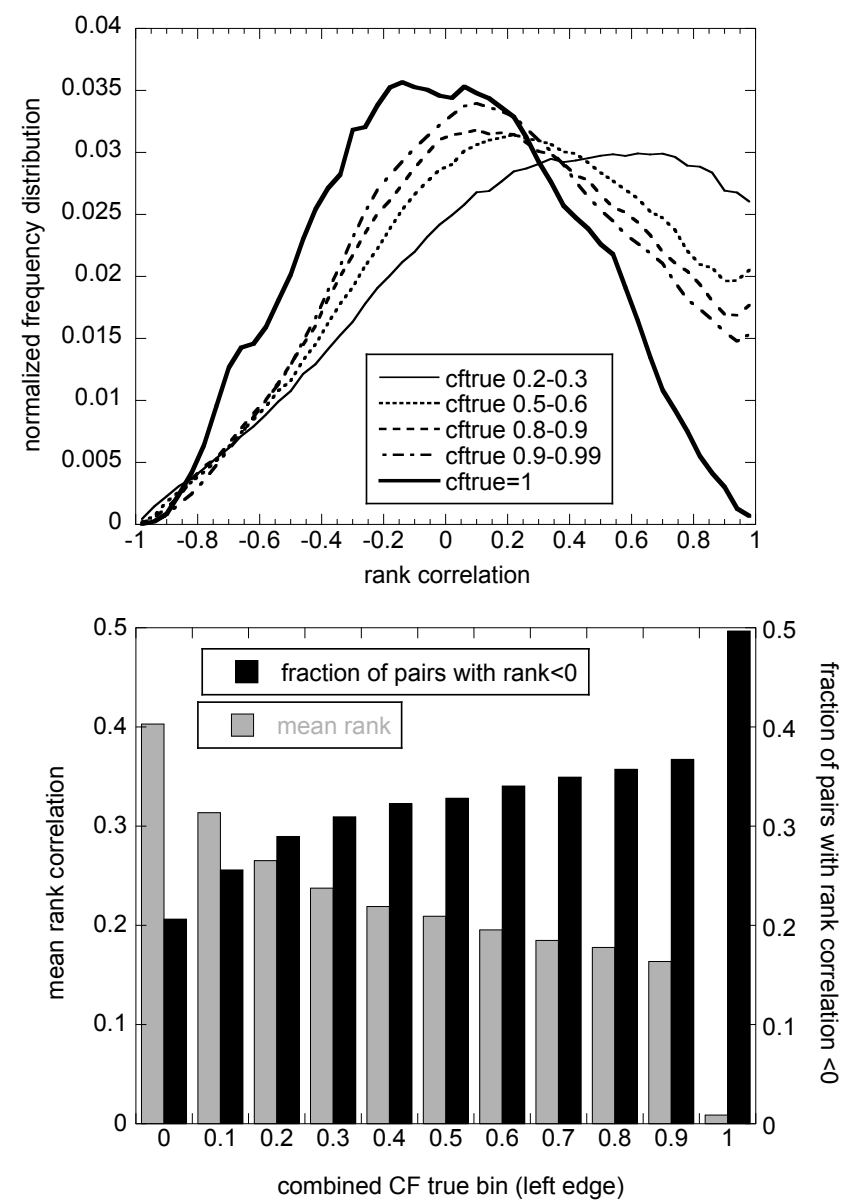

Fig. 6. (top) histograms of rank correlations for different bins of true combined cloud fraction calculated from layer pairs taken at every possible separation distance within $150 \mathrm{~km}$ segment sizes; (bottom) ensemble-averaged rank correlations and fraction of negative rank correlations within true combined cloud fraction bins from the same dataset used for the top panel. The x-axis labels in the lower panel indicate the left edge of the bin. For example 0.1 refers to the interval $[0.1,0.2)$, and the last bin is for a combined cloud fraction exactly equal to 1 .

calculation). We create two types of plots: one showing the frequency distribution of rank correlations for different bins of combined true cloud fraction or alpha, and one showing the ensemble mean ranks and fraction of negative mean ranks for those bins. The second type of plot essentially summarizes two features, the mean and the cumulative frequency up to zero rank correlation, found in plots of the first type, but for more bins than were practical to display in those plots.

The plots discussed above are shown in Figs. 6 and 7. Figure 6 suggests that when the combined cloud fraction of the layers is 1 the probability distribution of rank correlations is almost perfectly symmetric around zero and yields a nearzero mean rank. This is an interesting result that defies an obvious explanation. Combined cloud fractions of exactly 1 can occur only for overlap smaller than random, i.e., for some
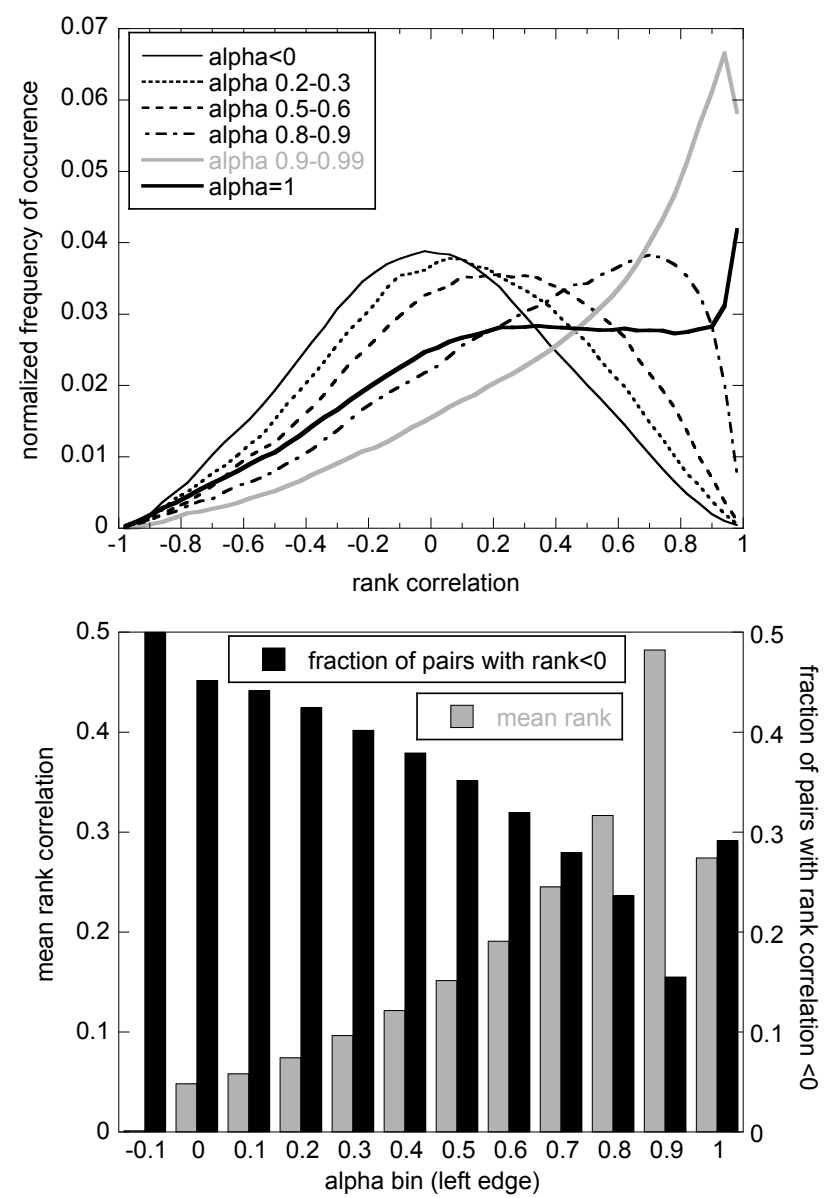

Fig. 7. As in Fig. 6, but for bins of overlap parameter alpha.

degree of minimum overlap. So it would be tempting to infer that so-called minimal overlap, which implies a smaller overlapped fraction, tends to be associated with zero mean rank correlation, but with a large amount of noise from ranks of either sign due to the small overlapped sample size.

However, there are significant and hitherto undiscussed interpretation issues that may be appropriate to raise here. Up until this point we have been treating alpha and rank correlation on a somewhat equal footing. In fact, however, they are quite different - rank correlation is a fairly robust statistical property based on a typically large number of rank pairs in the overlapped portion of the two layers. Alpha, by contrast, for a particular layer pair is based on only the two layer cloud fractions, and is not a statistically robust quantity unless averaged over an ensemble of many segments or unless the single segment in which it is evaluated is large compared to the horizontal length scale $l_{h}$ over which individual clouds in each layer become statistically uncorrelated. In other words, while single segment $\alpha\left(z_{1}, z_{2}\right)$ values of 1,0 and $<0$ do have specific meanings for the segment in terms of cloud overlap (maximum, random and some degree of minimum overlap) they imply little about the respective largescale statistical overlap of the two cloud layers over a large 
number of segments, unless the segment is large enough to contain many dynamically independent cloud samples. This is presumably why the standard deviations of alpha in Fig. 1 are so much larger and more variable than the respective rank correlation values of Fig. 2.

Now, let us apply this thinking to the example of $100 \%$ combined cloud fraction discussed above. Such a case implies $\alpha\left(z_{1}, z_{2}\right)<0$ (some degree of minimal overlap), however, two completely uncorrelated cloud layers (in the largescale sense) can frequently produce cases of $100 \%$ combined cloud cover in segments that are not large compared to $l_{h}$. In fact, the greater the individual layer cloud fractions, the greater the likelihood of this. Thus the $100 \%$ combined cloud fraction bin will be a "degenerate bin" that mixes many segments of large-scale uncorrelated layers with perhaps occasional segments of large-scale minimally overlapped layers. If these uncorrelated cases dominate, as they appear to, then it is not surprising that the condensate rank correlations within the bin are near zero in the mean. In this case, "minimal overlap" is likely to be a false designation, since the alphas are all single segment values.

Returning to Fig. 6, a progressive shift to fewer negative and greater mean rank correlations occurs when the combined cloud fractions become smaller, i.e., when the overlap becomes closer to maximum and the individual cloud fractions are also small. One possible explanation is a transition from large scale cloudiness (with large cloud fractions in either or both layers, yielding a large combined fraction, but from layers that can be quite unrelated) to convective clouds (typically of small cloud fractions, but of large vertical extent). Note that the 0.9-0.99 combined cloud fraction bin is quite distinct from the overcast bin in terms of its rank correlations. Within this bin, random cloud fraction overlap is possible, and positive ranks occur about $62 \%$ of the time. By the time the combined cloud fraction is between 0.01 and 0.1 about $80 \%$ of the rank correlations are positive.

Figure 7 is consistent with the above picture, since as it was explained earlier, the combined cloud fraction and alpha are not independent. For negative alpha the distribution of rank correlations is again almost perfectly symmetric around zero, and results in an almost exact zero mean rank correlation. As cloud fraction overlap shifts from random to maximum the distributions become progressively more negatively skewed and produce higher mean ranks until exact maximum overlap $(\alpha=1)$ is reached. For that bin the number of negative rank correlations goes up again and the value of the mean goes down, making it very distinct from the 0.9-0.99 alpha bin (near-maximum overlap) which contains the largest mean rank, larger than any mean rank in Fig. 6. Bear in mind that the $\alpha=1$ bin does not necessarily contain only small combined cloud fractions, so it should not be associated with any particular true combined cloud fraction bin in Fig. 6. A large value of alpha simply suggests that the probability of a small combined cloud fraction is statistically higher.

\section{Discussion of modeling implications}

We have presented an analysis of cloud overlap characteristics at a mid-latitude observational facility based on retrievals of cloud condensate from a millimeter cloud radar assisted by a suite of other ground instruments. The temporal (horizontal in an Eulerian sense) and vertical resolution of the data, at $10 \mathrm{~s}$ and $45 \mathrm{~m}$, respectively, are the highest ever used to study this problem. The two facets of overlap investigated were cloud fraction overlap (previously examined at the same site with coarser resolution datasets by Mace and BensonTroth, 2002; Naud et al., 2008) and the overlap of horizontal distributions of condendsate, which has never been previously examined with a dataset of this type. Besides the cloud fraction overlap parameter alpha and the rank correlation coefficient, the degree of proximity to random and maximum overlap was also expressed in terms of decorrelation lengths, a convenient scalar parameter equal to the e-folding length when the overlap parameter is assumed to decay exponentially with separation distance. Our findings regarding cloud fraction overlap, whether expressed in terms of alpha or its decorrelation length, reaffirm previous results with respect to seasonal variations and dependence on domain size, namely that overlap tends to be more maximum for summer months and larger domains. The same dependence is found for rank correlation, albeit significantly weaker, a behaviour not previously known. We sought to gain further insight into overlap parameter dependencies by examining differences in mean values for fixed separation distances within different layers of the atmospheric column, and by searching for possible systematic relationships between alpha and rank correlation. These efforts revealed that for the same separation distance the overlap parameters are significantly different at the various atmospheric layers, and that random cloud fraction overlap is generally associated with more random correlations of relative condensate strength.

The question that naturally arises is whether any of the above has practical implications. If one wants to create 2-D $\mathrm{X}-\mathrm{Z}$ distributions of condensate (a second horizontal dimension is irrelevant for fields with no predefined horizontal spatial coherence) starting from profiles of cloud fraction and the mean and variance of cloud condensate, overlap rules must be established. Our paper contains information about these overlap rules. Obviously, an extension to a global dataset is desirable, and the combined CloudSat/CALIPSO dataset may be of significant help in this regard (e.g., Mace et al., 2010). Also, a measure of whether overlap has been realistically and successfully implemented is necessary. A straightforward avenue of future research is to adopt the inverse exponential model and express overlap in terms of decorrelation lengths. Our dataset has shown that negative values for the overlap parameters are too frequent for the exponential framework to be consistently credible, but it may work adequately if the impact of replacing negative values with zero is small. Then there is the question what value of decorrelation 
length to use. Should the median of individual decorrelation lengths (derived from individual data segments) be used as in Barker (2008b)? Would a modified definition that yields an "effective" decorrelation length where the additional constraint of matching segment-level total cloud fractions is imposed (Barker, 2008a) be better? Or should the decorrelation length as derived in this work be used, namely from fits to ensemble-averaged profiles of alpha and rank correlation?

A research path may be available to help address these questions (e.g., see Barker 2008a, b). It essentially entails using the profiles of cloud fraction and the first two moments of condensate for each data segment, assuming a probability distribution function for the condensate, and reconstructing the cloud fields using either a single decorrelation length from average overlap parameter profiles or individual decorrelation lengths derived at the segment level, with a cloud generator of the type introduced by Räisänen et al. (2004). The appropriateness of the inverse-exponential model and of the proper decorrelation lengths can be tested by comparing: (a) cloud statistics (total cloud fraction or cumulative profiles of cloud fraction exposed to space and moments of water path) between the original and reconstructed cloud fields and (b) radiation flux and heating rates corresponding to the original and reconstructed cloud fields. Radiative comparisons of the latter type will be facilitated in the near future by the availability of the Radiatively Important Properties Best Estimate (RIPBE) evaluation product a final version of which is set to be released soon for the SGP ACRF site (S. McFarlane, personal communication, 2011). RIPBE relies for its cloud specification on the same MICROBASE dataset we use for our overlap analysis (albeit at a lower 1 minute temporal resolution), while also including all other atmospheric (temperature and water vapor profiles, aerosol loading, etc.) and surface (spectral albedo) variables that are required for full broadband radiative transfer calculations. Such a validation from both the cloud and radiation statistics perspectives is in our future plans.

Acknowledgements. The authors gratefully acknowledge support by the US Department of Energy, Office of Science, Office of Biological and Environmental Research, Environmental Sciences Division as part of the ARM program under grant DE-FG0207ER64354, and by the NASA Modeling Analysis and Prediction and CloudSat/CALIPSO Science Team Recompete programs managed by David Considine. We would like to thank Mike Jensen and Maureen Dunn of Brookhaven National Lab for generating and providing the MICROBASE data used in our analysis.

Edited by: A. Nenes

\section{References}

Barker, H. W.: Overlap of fractional cloud for radiation calculations in GCMs: A global analysis using CloudSat and CALIPSO data, J. Geophys. Res., 113, D00A01, doi:10.1029/2007JD009677, 2008 a.

Barker, H. W.: Representing cloud overlap with an effective decorrelation length: An assessment using CloudSat and CALIPSO data, J. Geophys. Res., 113, D24205, doi:10.1029/2008JD010391, 2008b.

Clothiaux, E. E., Ackermann, T. P., Mace, G. C., Moran, K. P., Marchand, R. T., Miller, M. A., and Martner, B. E.: Objective determination of cloud heights and radar reflectivities using a combination of active remote sensors at the ARM CART sites, J. Appl. Meteor., 39, 645-665, 2000.

Hogan, R. J. and Illingworth, A. J.: Deriving cloud overlap statistics from radar, Q. J. Roy. Meteor. Soc., 126, 2903-2909, 2000.

Hogan, R. J. and Illingworth, A. J.: Parameterizing ice cloud inhomogeneity and the overlap of inhomogeneities using cloud radar data, J. Atmos. Sci., 60, 756-767, 2003.

Liao, L. and Sassen, K.: Investigation of relationships between Kaband radar reflectivity and ice and water content, Atmos. Res., 34, 231-248, 1994.

Liu, C. L. and Illingworth, A.: Toward more accurate retrievals of ice water content from radar measurements of clouds, J. Appl. Meteor., 39, 1130-1146, 2000.

Mace, G. G. and Benson-Troth, S.: Cloud layer overlap characteristics derived from long-term cloud radar data, J. Climate, 15, 2505-2515, 2002.

Mace, G. G., Zhang Q., Vaughan M., Marchand R., Stephens G., Trepte C., and Winker D.: A description of hydrometeor layer occurrence statistics derived from the first year of merged Cloudsat and CALIPSO data, J. Geophys. Res., 114, D00A26, doi:10.1029/2007JD009755, 2010.

Miller, M. A., Johnson, K. L., Troyan, D. T., Clothiaux, E. E., Mlawer E. J., Mace G. G.: ARM Value-Added Cloud Products: Description and Status, in: Proceedings of the 13th ARM Science Team Meeting, US Department of Energy, Washington, DC, available at: http://www.arm.gov/publications/proceedings/ conf13/extended_abs/miller-ma.pdf, 2003.

Naud, C. M., Del Genio A., Mace G. G., Benson S., Clothiaux E. E., and Kollias, P.: Impact of dynamics and atmospheric state on cloud vertical overlap, J. Climate, 21, 1758-1770, doi:10.1175/2007JCLI1828.1, 2008.

Norris, P. M., Oreopoulos L., Hou, A. Y., Tao, W. K., and Zeng, X.: Representation of 3-D heterogeneous cloud fields using copulas: Theory for water clouds, Q. J. Roy. Meteor. Soc., 134, 18431864, 2008.

Oreopoulos, L. and Khairoutdinov, M.: Overlap properties of clouds generated by a cloud-resolving model, J. Geophys. Res., 108(D15), 4479, doi:10.1029/2002JD003329, 2003.

Press, W. H., Teukolsky, S. A., Vetterling, W. T., and Flannery, B. P.: Numerical recipes in Fortran 77, the art of scientific computing, 2nd ed., Cambridge University Press, 933 pp., 1992.

Pincus, R., Barker, H. W., and Morcrette, J. J: A fast, flexible, approximate technique for computing radiative transfer in inhomogeneous cloud fields, J. Geophys. Res., 108 (D13), 4376, doi:10.1029/2002JD003322, 2003.

Pincus, R., Hannay, C., Klein, S. A., Xu, K.-M., and Hemler, R.: Overlap assumptions for assumed probability distribution 
L. Oreopoulos and P. M. Norris: An analysis of cloud overlap at a midlatitude atmospheric observation facility

function cloud schemes in large-scale models, J. Geophys. Res., 110, D15S09, doi:10.1029/2004jd005100, 2005.

Räisänen, P., Barker, H. W., Khairoutdinov M., Li, J., and Randall, D. A.: Stochastic generation of subgrid-scale cloudy columns for large-scale models, Q. J. Roy. Meteor. Soc., 130, 2047-2067, 2004.
Shonk, J. K. P., Hogan, R. J., Edwards, J. M., and Mace, G. G.: Effect of improving representation of horizontal and vertical cloud structure on the Earth's global radiation budget. Part I: Review and parametrization, Q. J. Roy. Meteor. Soc., 136, 1191-1204, doi:10.1002/qj.647, 2010. 